\title{
A Conceptual Model for Privacy Policies with Consent and Revocation Requirements
}

\author{
Marco Casassa Mont ${ }^{1}$, Siani Pearson ${ }^{1}$, Sadie Creese ${ }^{2}$, \\ Michael Goldsmith ${ }^{2}$, and Nick Papanikolaou ${ }^{1}$ \\ ${ }^{1}$ Cloud and Security Lab, HP Labs, Bristol, UK \\ ${ }^{2}$ International Digital Laboratory, University of Warwick, Coventry, UK \\ \{marco.casassa-mont, siani.pearson, nick.papanikolaou\}@hp.com, \\ \{S.Creese, M.H.Goldsmith\}@warwick.ac.uk
}

\begin{abstract}
This paper proposes a conceptual model for privacy policies that takes into account privacy requirements arising from different stakeholders, with legal, business and technical backgrounds. Current approaches to privacy management are either high-level, enforcing privacy of personal data using legal compliance, risk and impact assessments, or low-level, focusing on the technical implementation of access controls to personal data held by an enterprise. High-level approaches tend to address privacy as an afterthought in ordinary business practice, and involve ad hoc enforcement practices; low-level approaches often leave out important legal and business considerations focusing solely on technical management of privacy policies. Hence, neither is a panacea and the low level approaches are often not adopted in real environments. Our conceptual model provides a means to express privacy policy requirements as well as users' privacy preferences. It enables structured reasoning regarding containment and implementation between various policies at the high level, and enables easy traceability into the low-level policy implementations. Thus it offers a means to reason about correctness that links low-level privacy management mechanisms to stakeholder requirements, thereby encouraging exploitation of the low-level methods. We also present the notion of a consent and revocation policy. A consent and revocation policy is different from a privacy policy in that it defines not enterprise practices with regards to personal data, but more specifically, for each item of personal data held by an enterprise, what consent preferences a user may express and to what degree, and in what ways he or she can revoke their personal data. This builds on earlier work on defining the different forms of revocation for personal data, and on formal models of consent and revocation processes. The work and approach discussed in this paper is currently carried out in the context of the UK collaborative project EnCoRe (Ensuring Consent and Revocation).
\end{abstract}

\section{Introduction}

Enterprises manage and administer huge sets of personal data which are collected as part of normal business practice. This process is complex and involves meeting a wide range of requirements, including the need to satisfy data protection and privacy laws, as well as any service requirements made by the enterprise or the consumer. Often such requirements are captured in the form of a policy or policies. However, there is not yet 
a unified view of the many and varied approaches to policy description and enforcement used in an enterprise. This makes it hard to guarantee that the combination of the various implementations does indeed meet all the requirements being made of the enterprise and is aligned with the law. Furthermore, the process of assessing this alignment is subject to human error.

In general there are two extreme approaches to management and enforcement of privacy policies. There is firstly a pragmatic approach, driven mainly by risk assessment and risk management and tailored to current business practices. It involves identifying suitable high level policies and points to act on, but then typically requires the deployment of pragmatic control points, which are very dependent on the specific scenario/environment. The control points enforcing policies are often hardcoded within applications and services in an ad hoc way, and so cannot easily be reused in different scenarios and organisational contexts - but this remains the norm in business practice today. Secondly, frequently research in this space tends to focus instead on a purely technical approach and narrowly propose yet another language or formal model for security, access control or obligation policies without taking into account legal, business and operational requirements. Hence, related policy languages might be too generic or detached from real requirements; often these languages and models are of interest to the research community but seldom widely adopted in real environments. We believe that there is a major gap between the two approaches and that there is a unique opportunity to combine aspects of each and provide mechanisms to bridge the two.

Our approach is to develop a conceptual model rich enough to describe high-level policies typically expressed in natural language, and structured to support their refinement and mapping into low-level technical policies for practical enforcement in an information system. In the EnCoRe (Ensuring Consent and Revocation) project ${ }^{1}$, we are exploring this approach while specifically focusing on an important aspect of privacy: the management of data subjects' (users') preferences with regard to the handling of their personal data. In EnCoRe such preferences actually equate to expressions of consent and revocation relating to rights to handle and process personal data. EnCoRe is exploring and demonstrating ways of handling consent and revocation policies in three key areas affecting people: enterprise data management, biobanks and assisted living.

While privacy policies mostly describe an enterprise's personal data handling practices, there is no commonly accepted way of stating clearly to customers whether (and which) mechanisms for granting and revoking consent for personal data are provided. In other words, what is desirable is a description of exactly which controls an enterprise provides to its customers. In this paper we call such a description a consent and revocation policy.

There is good reason for consent and revocation policies to be expressed in a machine-readable form, in a manner analogous to website privacy policies $[8,26]$, for then enforcement can be fully automated; our aim here is not to define the syntax of such policies, rather their structure and general characteristics.

Consent and revocation appear in many different forms, and the relationship between the two concepts is rather subtle. For instance, they are not symmetric: it is possible for an individual to revoke personal data for which consent has not been given (in Section 6 we will refer to this as consentless revocation). Consent itself is not a simple

${ }^{1}$ See http: / / www. encore-project.info 
yes/no ("store this data" or "do not store this data"), but has many subtly different gradations, depending on the type of data it refers to.

\section{Policy Layers and Dependencies in Organisations}

Organisations need to cope with a variety of policies and constraints that emerge from many different sources, including legislation (national and international), societal expectations, business requirements and (where appropriate) individual preferences expressed by users and customers. We concern ourselves here specifically with those policies relating to the handling of personal data and privacy.

Whilst privacy requirements are in general context dependent, we believe that there are a core set of privacy concepts which are common and underpin the various controls designed to deliver privacy against this varying set of requirements. They are, in effect, a tool box which can be utilised depending upon the unique requirements of the situation. But, due to the heterogeneity of the policies and of the languages used to express privacy requirements, it may not be always obvious which core privacy concepts are actually being utilised. However, if these are clearly identified, we will be able to better formalise and classify privacy-related policies, laws and technical solutions enabling a simplification and easier re-use of the technologies and methodologies designed to implement such policies. Further, the extraction of such core privacy concepts might make it easier to compare privacy legislation with the technical implementation of privacy constraints in a product.

We consider policies to fit within a layer model which in itself represents a hierarchy of policies. In this model, high-level policies express general requirements and rights that individuals have with regards to their privacy, as embodied typically in the law, business and regulatory requirements as they contain general constraints on business practice with regards to personal data. At the highest level of the classification, there is a set of requirements which are set out by international agreements and directives, such as the European Data Protection Directive or the EU Safe Harbour agreement. Further, many countries have national data protection legislation, such as the Data Protection Act 1998 in the UK, or HIPAA, GLBA, SB 1386, COPPA and various State Breach laws in US. With regards to regulation in particular, there are export and transborder flow restrictions on personal data that need to be enforced. Privacy laws and regulations constitute the topmost layers of policy hierarchy regarding personal data with which an enterprise must comply. Such policies are often expressed in natural language as is typically the case with related data subjects' preferences.

At this high level of abstraction, security requirements may include adherence to the Sarbanes-Oxley Act (SOX) for financial reporting, or the PCI Data Security Standard (DSS). These may be refined to a set of policies at a lower level. Similarly, business requirements include contractual obligations, information lifecycle policies and the enterprise's own internal guidelines. All of the above influence how personal data is collected, stored and administered.

Low-level policies are those which describe how privacy requirements are implemented in a particular piece of hardware, or in software that handles personal data. Such policies comprise detailed conditions on how particular data may be handled within a system: often these are just statements prohibiting particular accesses of the data, in which case they are referred to as access control policies.

At lower levels there are various operational and technical policies that are machine readable and enforceable by policy management frameworks, e.g. [1,4,6,19]. Among 


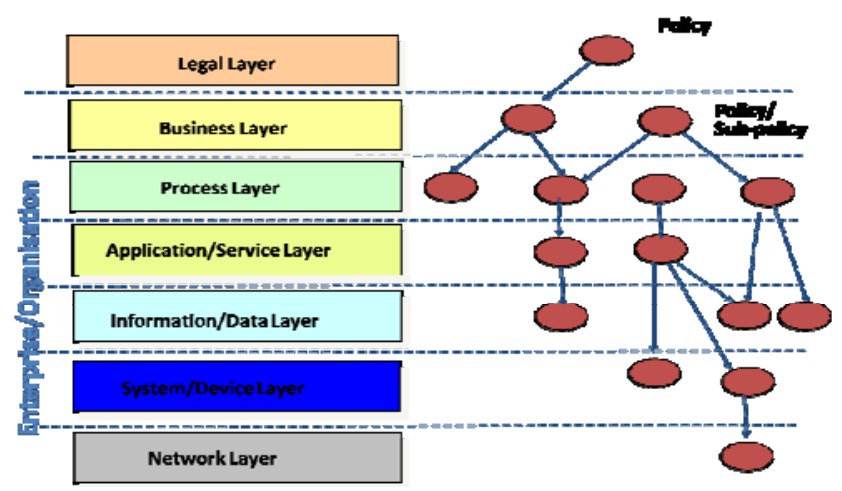

Fig. 1. The different layers in which privacy policies are implemented

these there will be policies expressing how a particular class of data is to be treated and these are only specific to the data, not to the system implementing the policies. Even more low level will be policies that are system-specific, and cannot be ported directly to other privacy-preserving platforms. For instance, policies specific to a particular health information system may contain specialized fields that do not exist in other similar systems. Figure 1 below is a diagrammatic representation of the different layers within which privacy policies are implemented. High level policies relate to the layers from the "Application/Service Layer" and above shown in Figure 1, while the layers below can be considered as low level policies. The preferences of a data subject are high level policies that lie between the Business Layer and the Legal Layer.

What is clear from the above analysis is that the origins of privacy requirements which an enterprise has to meet are very diverse, and they arise at many different levels of abstraction. In an ideal world, lower level policies should always be the result of refinements, or special cases, of the higher level ones. In the real world, high-level requirements change over time. Data subjects and data controllers (service providers) exercise choices relating to their preferences and risk appetites. This makes it impossible for a system to always be a correct refinement of requirements, as it will take time for choices to be implemented. It will be for the data subjects to decide whether they are being offered appropriate service levels regarding the response to their choices, and for service providers to determine what level of guarantee is appropriate for their business model. Law and regulation will also evolve over time, although much more slowly and in a manner which should give enterprises sufficient time to ensure that they are addressing (or at least attempting to address) changes to related policy requirements. Privacy requirements are so heterogeneous that is not always possible to treat them consistently, and yet it is necessary to ensure that all these assorted requirements are simultaneously met for the correct functioning of society.

A key assumption in our definition of a hierarchy, as opposed to a loose grouping of policies by theme and/or level of detail, is that there is a relation of containment between the different levels described. (In any enterprise, it should be possible to automatically check that this containment holds.) It should often be the case that higher-level policies express requirements that should be made more explicit (refined) in lower-level ones. In that sense, higher level policies contain requirements expressed 


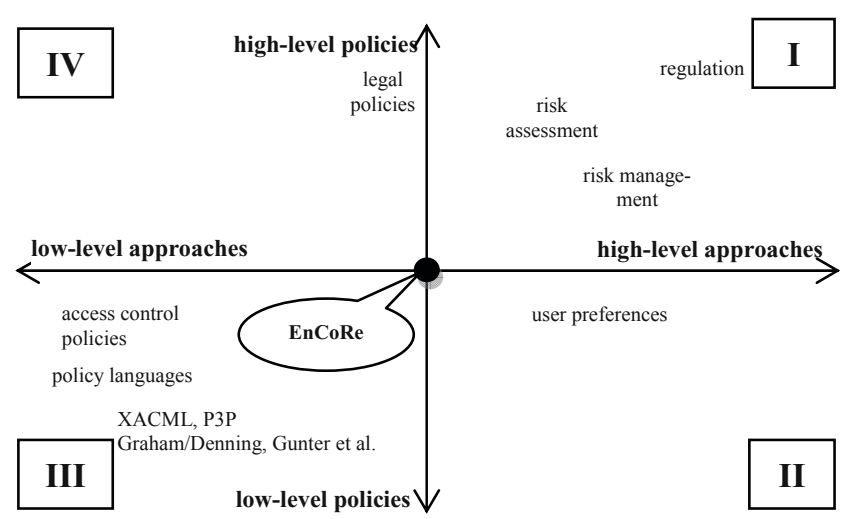

Fig. 2. Policy layers versus description and enforcement approaches

at the lower levels, albeit in a more abstract or generic form. This justifies their placement at the upper level of the hierarchy. The more formally a policy can be expressed, the more chance we have of creating automatic enforcement mechanisms reusable technology. However, there will always be policies which are, by design, open to interpretation and requiring human intervention.

One might classify current research in privacy policy description, management and enforcement using Figure 2. The vertical axis represents the varying levels at which policies are expressed at ranging from high-level (legal, regulatory) to low level (security/access control policies and user preferences). The horizontal axis characterises the degree to which policies are formalised, ranging from natural language to machine readable formats.

A significant amount of research falls into quadrant III. This is no surprise as the development of policy languages goes hand in hand with the development of machine readable descriptions of low-level technical policies.

It is evident from the figure that there are many other viewpoints and levels of abstraction that are of concern in policy management, and that there is scope for much work in the areas labelled as quadrants I, II and IV.

Quadrants II and III pertain to the low-level policy implementations and the degree to which they directly implement requirements expressed in natural language versus machine readable formats. Specifically, we see that there is a research opportunity providing a link between natural language requirements and policy implementations (II), whereas those requirements expressed directly into machine readable formats are a good fit (III). In reality we expect most requirements to begin life in II and that system implementations find ways of pulling these into III. Our conceptual model is designed to provide a formal framework within which traceability between requirements expressed in II can be linked to corresponding requirements in III.

Quadrants I and IV pertain to mapping of high-level policies directly into machine readable formats. Here the research question is to understand just how much of this kind of policy is ambiguous and requires context-dependent human intervention. We focus on ensuring that our conceptual model is rich enough to describe all of these high-level policies, so that where core privacy requirements can be identified we can directly map into formalised machine readable formats to support technology controls. 
The specific area of management of privacy policies, security constraints, consent and revocation [2] is of particular interest because it is at the intersection of legislation, user requirements and management of privacy and security technical policies within and across organisations. What is particularly desirable is to devise an intermediate representation of policies that embodies high-level requirements whilst being directly translatable to (potentially existing) low-level policies or access control languages such as XACML [5], EPAL, P3P [3], P-RBAC [6], PRIME [24] and the like. Such a representation should not be tied to a particular implementation language.

It may be argued that our definition of a hierarchy for privacy policies is arbitrary, as the level of detail contained in privacy-related documents, from international legislation down to business and regulatory policies, varies substantially by domain of application. It is the case that how the hierarchy is defined is heavily context-dependent. Our classification is based on research within EnCoRe, taking into consideration privacy requirements coming from a variety of sources (including legal, social and technical ones) related to the following scenarios:

- Employee data held within an enterprise

- Biobanks

- Assisted living

We expect that these case studies will guide our intuition regarding a core, common set of privacy requirements, and hence suggest the evolution of our conceptual model. We note here the previous work by Samarati et al. [27] which treats further the divide between high-level policies and low-level enforcement mechanisms.

\section{Towards a Conceptual Model}

The examples of policy rules we have given so far demonstrate several different forms that privacy requirements take in real business applications. It is desirable to be able to automatically enforce as many policy rules as possible; for this, a machinereadable representation of the different forms of requirements is necessary. However, the purpose of a conceptual model is to provide a representation that enables human systematic reasoning about policies while at the same time being convertible into machine-readable code.

A conceptual model defined in a strict mathematical way would have the benefit of being completely unambiguous, but it would likely be too restrictive, especially if it is intended to capture privacy laws and regulations. A more flexible approach would be to describe privacy requirements in a semi-formal manner. One can be very systematic and formal about the purpose of different policy rules, and in terms of syntax it should be possible to identify the main patterns of usage that occur in privacy requirements.

To illustrate this last point: above we identified policy rules as typically having the structure if $<$ some condition is met $>$ then $<$ action $_{1}>$ else $<$ action $_{2}>$ (the else part being optional). The syntax and semantics of the conditions and actions allowed in such rules are essentially informal. However, our analysis shows that there are at least the following core set of rule types:

- notification rules: such rules describe when and how data subjects should be notified regarding accesses, uses, and transfers of their personal data. Such rules appear in low-level policies - forcing an implemented system to send email or 
instant messages when a condition is triggered - as well as in high-level policies and legislation: the Data Protection Act, for example, specifies that data subjects can make subject access requests (SARs), forcing a data controller to notify them of any data held about them, for which purposes, and with whom this data has been shared.

- access control rules: such rules specify who can access data held by an enterprise; for instance, personal data about employees should only be available to the HR department and to the employees (on an individual basis). The standard if-then-else structure of access control rules was first identified by Bonatti et al. [28].

- update/creation rules: these rules express who is permitted to modify personal data that is held, and under which conditions. The right to update data or even create new data is usually reserved for the data subject, and certainly such a right exists in legislation. Rules specifying who can perform such changes to data held typically take into account the role of the parties making them (cf. role-based access control).

- protection rules: there will be rules specifying protections on particular data, usually protections of a technical nature, such as encryption. These are most easily described in technical, low-level privacy policies, since the parameters and algorithm for encryption can be explicitly defined; however, requirements for encryption are increasingly found in privacy regulation and company privacy policies.

- obligation rules: these are rules specifying a data controller's commitment to implement a future policy decision, or to apply a particular rule (e.g. forcing the deletion of data at a particular date). Obligation management in information systems is a subject in its own right, and we are still studying this aspect.

These rule types are the essence of our conceptual model, and provide a natural means of expressing both high-level policies and setting requirements for low-level implementations. They enable the expression of actions associated with granting and revoking consent for the use of personal data.

\section{How Consent and Revocation Are Perceived by Different Stakeholders}

This section provides an informal overview of how the concepts of consent and revocation (and related policies) are perceived and dealt with by the key stakeholders, notably organisations, data subjects and regulators. The remaining part of the paper will then primarily focus on the data subjects' perspective.

Conceptually there are several layers of abstraction of policies, ranging from human readable high-level policies to semi-formal representations (which are actionable by humans while also being translatable into operational policies), to machinereadable policies that are not usually intended to be exposed to users. This also applies to consent and revocation policies, which specifically deal with the handling of personal and confidential data based on privacy preferences and constraints driven by data subjects and legislation.

Different stakeholders have different priorities and views of consent and revocation, as described below. 
Organisations. Organisations have a pragmatic view about consent and revocation. Incorporating these processes into their business practices requires effort and investment, esp. to provide necessary enforcement mechanisms; also potential liabilities, in case of failures, are introduced.

Because of this, it is common practice that these policies are represented in the form of opt-in or opt-out choices for end users - as these are simple to handle and not requiring complex enforcement mechanisms. In addition, consent and revocation policies are just a small part of the overall set of policies organisations need to take into account, for example business policies, security policies, compliance policies, etc.

Current legislation enables organisations, in some circumstances, to bypass the need to obtain consent (and revocation) from end-users, based on "fair usage". Of course organisations need to take into account the trade-off between carrying on with the current minimalistic data management practice and the increasing interest and willingness of end-users to obtain control over their data.

EnCoRe is exploring how to ease the pain in dealing with consent and revocation management and the enforcement of related policies hence hoping to shift organisational behaviour towards a more privacy-friendly approach.

Data Subjects (End-Users). From the point of view of a data subject, or end-user, if an enterprise provides consent and revocation controls, it increases that user's choice regarding how his or her personal data is handled. We are interested here in the point of view of the end-user, and in Section 7 we argue that an enterprise can define its own consent and revocation policy, as a way of informing end-users of the choices available to them.

Regulators. Regulators have been very active in providing laws and legislation concerned with the management (collection, processing and disclosure) of personal data. Laws are usually abstract and primarily focus on the circumstances in which consent might be necessary. On the other hand the concept of revocation is fuzzy and not properly addressed. As a consequence, policies that derive from these laws and legislation do not properly address these aspects and in particular meeting the expectations of the end-users.

\section{Forms of Consent and the Notion of a Consent Variable}

With regard to an individual's personal data, there are four principal things for which an enterprise or other data collector requires that individual's consent:

- collection of data,

- processing of data,

- sharing of data.

Collection of data refers to the initial process by which data is acquired and stored on the enterprise's information system. Processing includes any access of the data that has been collected and is characterised by a stated purpose (e.g. research, marketing, aggregation to derive average customer habits). Data may be shared - internally and externally (e.g. to third parties) so that it can be processed, often elsewhere than the site of data collection. 
Thus, consent may be defined as a wish for a datum $d$ to be collected, processed, shared, or any combination of the above. This definition is too coarse, however, for it does not account for subtleties such as these:

- It may be desired to restrict data collection so that it occurs only in one country, so that it is subject to that country's privacy legislation.

- It may be desired that consent lasts for only a fixed period of time.

- It may be desired to restrict processing of data so that it is used for only certain stated purposes.

- It may be desired that the data is shared with only particular parties, and to banish uses of the data by others.

Thus we claim that consent is parameterised by certain quantities referred to as consent variables. As examples we consider: time $t$ for which consent is granted, volume $v$ of data for which consent is granted, the set $S$ of allowed purposes (stated purposes for which consent is granted), the set $\Pi$ of parties who may access the data.

Thus, consent is fully determined when there are specified:

- the task for which consent is given (collection, processing, sharing, or any combination thereof)

- for this task, the values of the consent variables of interest.

From this one can extract a mathematical definition of consent and develop a hierarchy of its different forms. Formal models of consent and the attendant logic are addressed in our other work [26].

\section{Forms of Revocation}

Revocation corresponds to the withholding or withdrawal of consent. It is manifested in its simplest form as deletion of data, although there are many variations of revocation, as listed below (from [8]):

1. No Revocation At All: Personal data remains static, and once it has been disclosed, it is either physically impossible to revoke (how could ever revoke reputation) or prohibited for various reasons (e.g. law-enforcement, data from police's DNA database).

2. Deletion: Data are completely erased and cannot be retrieved or reconstituted in any way.

3. Revocation of Permissions to Process Data: Data subjects withdraw consent that would enable an enterprise to process or analyse their personal data for a specified purpose.

4. Revocation of Permissions for Third Party Dissemination: Data subjects withdraw consent that would enable an enterprise to disclose information to a third party.

5. Cascading Revocation is a variation on any of the above kinds of revocation, whereby the revocation is (recursively) passed on to any party to whom the data has been disclosed. Through this mechanism, data subjects are able to revoke data by only contacting the enterprise that they disclosed their data to originally. 
6. Consentless Revocation: Personal data for whose storage and dissemination no consent has been explicitly given by the user, but which may need to be revoked. Again, any of the fundamental types of revocation may be invoked. The need to revoke consentless data emerges mainly when a breach in privacy has occurred. In the italics below we describe a characteristic example of consentless revocation.

7. Delegated Revocation: This is a kind of revocation which is exercised by a person other than the individual concerned, such as an inheritor or parent/guardian.

8. Revocation of Identity (Anonymisation): Data subjects may be happy for personal data to be held for certain purposes so long as it is not linkable back to them personally. Anonymisation may be regarded as a variant of revocation, in that data subjects request a change to data held so that it is no longer personally identifiable.

These forms of revocation should be available as actions that individuals can perform on personal data held about them by an enterprise.

\section{Defining Consent and Revocation Policies}

Having enumerated and explained the main forms and variants of consent and revocation, we are in a position to define the notion of a consent and revocation policy, whose purpose is to enable an enterprise to inform customers of:

a) what consent is required of them for each data field, and

b) which revocation types are available to them should they wish to exercise control over their personal data.

Thus, a consent and revocation policy is defined over a set of data $D=\left\{\delta_{i}\right\}$ as a set of tuples

$$
\left\{\forall i:\left(\delta_{i}, c_{i}, p_{i}, d_{i}, R_{i}\right)\right\}
$$

where the $c_{i}, p_{i}, d_{i}$ define together a value of consent (specifying collection, processing and dissemination rights respectively), and $R_{i}$ is a set consisting of the names of allowed revocation types.

As an example, consider this policy over $D=\left\{\delta_{1}, \delta_{2}\right\}$ :

$$
\begin{gathered}
\left\{\left(\delta_{1}, c(30 \text { days }),-, d(10 \text { days }),\{2,3\}\right),\right. \\
\left.\left(\delta_{2},-, p(10 \text { days }),-,\{2\}\right)\right\}
\end{gathered}
$$

What this policy specifies, assuming the only consent variable of interest is the time for which data is held, is that consent is required to enable the collection of $\delta_{1}$ for 30 days and its dissemination for 10 days; furthermore, $\delta_{1}$ can be revoked using revocation types 2 (deletion) and 3 (revocation of permissions to process data). The example policy also states that consent to process $\delta_{2}$ for 10 days is required, and that deletion may be performed.

Note that the symbol - is used when one of the $c_{i}, p_{i}, d_{i}$ is omitted. Some further formalisation work is needed to make the definitions more rigorous, but we believe that the above presentation is sufficient for our purposes here.

An enterprise is likely to require stipulations on the minimum consent a customer can provide for each data field. In other words, rather than stating in a policy specific consent values that an enterprise needs from a customer, it may be desirable to specify a range of values. We regard this as a direction for further investigation. 


\section{Conclusions and Future Work}

We have discussed in this paper issues to do with the description, management and enforcement of policies in organisations. Specifically we highlighted the gap existing from a high-level approach to policies driven by risk and privacy impact assessment and low-level technical policies.

We strongly believe this gap needs to be filled to enable continuity of requirements and constraints across all these levels and enable proper enforcement of policies. To achieve this we proposed the adoption of a conceptual policy model, to enable reasoning and mapping of concepts at lower levels of abstraction.

We have in this paper explained the significance of consent and revocation preferences for personal data, and introduced the notion of a consent and revocation policy. We have described in detail the different aspects of the notions of consent and revocation respectively, and explained how an enterprise can define policies that make clear what options are available to a customer in terms of controlling his or her personal data.

Our future work will seek to validate and refine our conceptualisation of a policy hierarchy, specifically with a view to ensuring that our conceptual model for privacy policy is rich enough to cater for all needs. We will develop a complete conceptual model encompassing a formal syntax and semantics. We will also investigate the utility of the conceptual model by application to case studies, initially within the EnCoRe project. We hope to be able to identify core privacy properties across the case studies, which can be easily mapped into reusable low-level control mechanisms. We also hope that this will offer opportunities to simplify the human interfaces, and reduce the amount of human intervention required making it simpler and more cost effective.

There is much further work to be done on consent and revocation policies, most notably refining the definition of the $R_{i}$, so that the revocation types include parameters analogous to consent variables. In practical terms, we expect to link consent and revocation policies to a real-world access control language, such as XACML, so that enforcement of consent and revocation can be done programmatically. There are also considerations to do with the internal consistency of such policies, such as preventing conflicts or incompatibilities between the consent requested and the revocation types made available.

We believe that it is essential to incorporate consent and revocation controls in enterprise information systems that handle personal data, and that the deployment of consent and revocation policies across such systems is a useful means of ensuring that the privacy preferences of individuals will be respected.

\section{Acknowledgements and Notes}

We gratefully acknowledge the support of the EnCoRe project sponsors - TSB, EPSRC and ESRC. The EnCoRe project [5] is an interdisciplinary research project, undertaken collaboratively by UK industry and academia, and partially funded by the Technology Strategy Board (TP/12/NS/P0501A), the Engineering and Physical Sciences Research Council and the Economic and Social Research Council (EP/G002541/1). It is noted here that the affiliation of author NP has changed after the original version of this paper was presented at the workshop. 


\section{References}

[1] Mont, M.C.: On the Need to Explicitly Manage Privacy Obligation Policies as Part of Good Data Handling Practices. In: Proceedings of W3C Workshop on Languages for Privacy Policy Negotiation and Semantics-Driven Enforcement, Ispra, Italy, October 17-18 (2006)

[2] Mont, M.C., Pearson, S., Kounga, G., Shen, Y., Bramhall, P.: On the Management of Consent and Revocation in Enterprises: Setting the Context. Technical Report HPL-200949, HP Labs, Bristol (2009)

[3] Cranor, L., Dobbs, B., Egelman, S., Hogben, G., Humphrey, J., Langheinrich, M., Marchiori, M., Presler-Marshall, M., Reagle, J.M., Schunter, M., Stampley, D.A., Wenning, R.: The Platform for Privacy Preferences 1.1 (P3P1.1) Specification. World Wide Web Consortium Note NOTEP3P11-20061113 (2006)

[4] Mont, M.C., Thyne, R.: Privacy Policy Enforcement in Enterprises with Identity Management Solutions. In: PST 2006 (2006)

[5] OASIS, eXtensible Access Control Markup Language (XACML), http://www.oasis-open.org/committees/tc_home.php? wg_abbrev=xacml

[6] Ni, Q., Trombetta, A., Bertino, E., Lobo, J.: Privacy-aware role based access control. In: Proceedings of the 12th ACM Symposium on Access Control Models and Technologies, Sophia Antipolis, France, June 20-22, pp. 41-50. ACM, New York (2007)

[7] Ferrini, R., Bertino, E.: A Comprehensive Approach for Solving Policy Heterogeneity. In: ICEIS 2009 -Proceedings of the 11th International Conference on Enterprise Information Systems, Milan, Italy, May 6-10, pp. 63-68 (2009)

[8] Agrafiotis, I., Creese, S., Goldsmith, M., Papanikolaou, N.: Reaching for Informed Revocation: Shutting Off the Tap on Personal Data. In: Proceedings of Fifth International Summer School on Privacy and Identity Management for Life, Nice, France, September 7-11 (2009)

[9] IBM, The Enterprise Privacy Authorization Language (EPAL), EPAL specification, v1.2 (2004),

http://www. zurich.ibm.com/security/enterprise-privacy/epal/

[10] Vaniea, K., Karat, C., Gross, J.B., Karat, J., Brodie, C.: Evaluating assistance of natural language policy authoring. In: Proc. SOUPS 2008, vol. 337 (2008)

[11] IBM, REALM project,

http: / /www. zurich.ibm.com/security/publications /

$2006 /$ REALM-at-IRIS2006-20060217.pdf

[12] OASIS, eContracts Specification v1.0 (2007),

http: / /www. oasis-open.org/apps / org/workgroup/

legalxml-econtracts

[13] Travis, D., Breaux, T., Antón, A.: Analyzing Regulatory Rules for Privacy and Security Requirements. IEEE Transactions on Software Engineering 34(1), 5-20 (2008)

[14] W3C, The Platform for Privacy Preferences, v1.0 (2002), http: / / www.w3 . org/TR/P3P/

[15] Kenny, S., Borking, J.: The Value of Privacy Engineering. Journal of Information, Law and Technology (JILT) 1 (2002),

http://elj.warwick.ac.uk/jilt/02-/kenny.html

[16] Organization for Economic Co-operation and Development (OECD), Guidelines Governing the Protection of Privacy and Transborder Flow of Personal Data, OECD, Geneva (1980) 
[17] Borking, J.: Privacy Rules: A Steeple Chase for Systems Architects (2007),

http: / /www.w3 org/2006/07/privacy-ws / papers /

04-borking-rules/

[18] Cranor, L.: Web Privacy with P3P. O’Reilly \& Associates, Sebastopol (2002)

[19] Damianou, N., Dulay, N., Lupu, E., Sloman, M.: The Ponder Policy Specification Language (2001), http: //www-dse.doc.ic.ac.uk/research/policies/index.shtml

[20] PRIME, Privacy and Identity Management for Europe (2008), http: //www.prime-project.org.eu

[21] IBM: Sparcle project, http: //domino.research.ibm.com/comm/research_projects.nsf/ pages/sparcle.index.html

[22] The GRC-GRID, The Governance, Risk Management and Compliance Global Rules Information Database, http: / / www.grcroundtable.org/grc-grid.htm

[23] Archer: Compliance Management solution, http: / / www .archer-tech.com

[24] Pearson, S., Sander, T., Sharma, R.: A Privacy Management Tool for Global Outsourcing. In: Garcia-Alfaro, J., Navarro-Arribas, G., Cuppens-Boulahia, N., Roudier, Y. (eds.) DPM 2009. LNCS, vol. 5939. Springer, Heidelberg (2010)

[25] Ardagna, C.A., Cremonini, M., De Capitani di Vimercati, S., Samarati, P.: A PrivacyAware Access Control System. Journal of Computer Security, JCS (2008)

[26] Westin, A.: Privacy and Freedom. Athenaeum, New York (1967)

[27] Agrafiotis, I., Creese, S., Goldsmith, M., Papanikolaou, N.: The Logic of Consent and Revocation (2010) (submitted)

[28] Samarati, P., De Capitani di Vimercati, S.: Access Control: Policies, Models, and Mechanisms. In: Focardi, R., Gorrieri, R. (eds.) FOSAD 2000. LNCS, vol. 2171, p. 137. Springer, Heidelberg (2001)

[29] Bonatti, P., Damiani, E., De Capitani di Vimercati, S., Samarati, P.: An Access Control Model for Data Archives. In: Proc. of the 16th International Conference on Information Security, Paris, France (June 2001) 